\title{
Applications of Statistics to Medical Science, II Overview of Statistical Procedures for General Use
}

\author{
Hiroshi Watanabe \\ Department of Mathematics, Nippon Medical School
}

\begin{abstract}
Procedures of statistical analysis are reviewed to provide an overview of applications of statistics for general use. Topics that are dealt with are inference on a population, comparison of two populations with respect to means and probabilities, and multiple comparisons. This study is the second part of series in which we survey medical statistics. Arguments related to statistical associations and regressions will be made in subsequent papers.
\end{abstract}

(J Nippon Med Sch 2012; 79: 31-36)

Key words: statistical test, statistical inference

\section{Introduction}

In the previous work ${ }^{1}$, we discussed a conceptual framework of statistical tests and statistical inferences. The present paper intends to give an overview of applications of statistics for general use.

The aim of statistics is to draw inferences on populations from samples that are randomly chosen from populations. Statistical inference is made as point estimation, interval estimation, and a statistical test for population parameters. In this paper, we describe typical analysis procedures from these 3 viewpoints.

Textbooks $^{2.3}$ are rich sources of information on medical statistics. In particular, we find medical examples of the statistical procedures presented in this paper.

\section{Inference on a Population}

parameters of a population.

\section{Point Estimation}

Let $M$ be a population with mean $\mu$ and variance $\sigma^{2}$. To infer the values of $\mu$ and $\sigma^{2}$ from a sample $x_{1}$, $x_{2}, \cdots, x_{n}$ that are randomly chosen from $M$, we compute a sample mean $\bar{x}$ and an unbiased variance $v^{2}$ defined by

$$
\begin{gathered}
\bar{x}=\frac{1}{n} \sum_{i=1}^{n} x_{i}=\frac{1}{n}\left(x_{1}+x_{2}+\cdots+x_{n}\right), \\
v^{2}=\frac{1}{n-1} \sum_{i=1}^{n}\left(x_{i}-\bar{x}\right)^{2} .
\end{gathered}
$$

The expectation values of $\bar{x}$ and of $v^{2}$ are equal to $\mu$ and $\sigma^{2}$, respectively. Furthermore, if the sample size $n$ is large, values of $\bar{x}$ and of $v^{2}$ concentrate in the neighborhoods of $\mu$ and of $\sigma^{2}$, respectively. In this sense, we write

$$
\mu \approx \bar{x}, \sigma^{2} \approx v^{2} .
$$

This is the reason we use $\bar{x}$ and $v^{2}$ as estimators of $\mu$ and $\sigma^{2}$, respectively.

In this section, we describe how to infer

Correspondence to Hiroshi Watanabe, Department of Mathematics, Nippon Medical School, 2-297-2 Kosugi-cho,

Nakahara-ku, Kawasaki 211-0063, Japan

E-mail: watmath@nms.ac.jp

Journal Website (http://www.nms.ac.jp/jnms/) 
around the value of $\mu$. The magnitude of an error $E$ associated with this estimation is measured by (the square root of) the variance of $\bar{x}$. Because the variance of $\bar{x}$ is equal to $\sigma^{2} / n$ and $\sigma^{2}$ has the estimator $v^{2}$, the error $E$ is estimated by the statistic $v / \sqrt{n}$, which we call a standard error of $\bar{x}$ and write

$$
\mathrm{SE}(\bar{x})=\frac{v}{\sqrt{n}} .
$$

\section{Interval Estimation}

Let us assume that the population $M$ obeys a normal distribution. Then, the statistic

$$
t=\frac{\bar{x}-\mu}{\operatorname{SE}(\bar{x})}=\frac{\bar{x}-\mu}{v / \sqrt{n}}
$$

obeys a $t$ distribution with $n-1$ degrees of freedom. On the basis of this fact, we obtain an interval estimation for $\mu$ as follows. Denote the $(1-\alpha / 2) \times$ 100 th percentile of the $t$ distribution with $n-1$ degrees of freedom by $t_{n-1}(1-\alpha / 2)$. Then, the confidence interval of $\mu$ with confidence level $1-\alpha$ is given by

$$
\bar{x}-\delta<\mu<\bar{x}+\delta
$$

with

$$
\delta=t_{n-1}(1-\alpha / 2) \operatorname{SE}(\bar{x}),
$$

which follows from the bound $|t|<t_{n-1}(1-\alpha / 2)$.

\section{Statistical Test}

Let $\mu_{0}$ be an arbitrary constant. Then, the null hypothesis $H_{0}: \mu=\mu_{0}$ is tested by means of the confidence interval (6). A test with significance level $\alpha$ is implemented by the following rule:

Accept $H_{0}$ if and only if $\mu=\mu_{0}$ belongs to the interval (6), i.e., if and only if $\left|\bar{x}-\mu_{0}\right|<\delta$ holds,

where $\delta$ is defined by (7). This test is referred to as a one-sample $t$ test.

Remarks. 1) A one-sample $t$ test is, in fact, available in the situation where the distribution of the population might slightly deviate from a normal distribution. We refer to this property of the onesample $t$ test as robustness. However, if the distribution is far from being normal and the sample size is not large, we should use nonparametric statistical methods, such as the Wilcoxon signed-rank test, for the null hypothesis that the (population) median is $\mu_{0}$.

2) The one-sample $t$ test for the null hypothesis $\mu$ $=0$ is especially important for comparison of paired samples. See section 3.

3) The definition (5) of $t$ has the form "(estimator parameter)/SE”. We shall see later further examples of this form.

4) Interval estimation of $\sigma^{2}$ is possible based on the fact that $(n-1) v^{2} / \sigma^{2}$ obeys a chi-square distribution with $n-1$ degrees of freedom.

\section{Comparison of Two Populations: Means}

In this section, we describe how to compare means of two populations.

\section{Point Estimation}

Let $M_{x}$ and $M_{y}$ be populations with means $\mu_{x}$ and $\mu_{y}$, respectively, and with the same variance

$$
\sigma_{x}^{2}=\sigma_{y}^{2}
$$

To compare means, we estimate the difference $\mu_{x}$ - $\mu_{y}$. Let $x_{1}, x_{2}, \cdots, x_{m}$ and $y_{1}, y_{2}, \cdots, y_{n}$ be samples randomly chosen from $M_{x}$ and $M_{y}$, respectively. Denote their sample means by $\bar{x}$ and $\bar{y}$, respectively, and their unbiased variances by $v_{x}^{2}$ and $v_{y}^{2}$, respectively. The difference $\mu_{x}-\mu_{y}$ has the estimator $\bar{x}-\bar{y}$ and the squared standard error $\mathrm{SE}(\bar{x}-\bar{y})^{2}$ of $\bar{x}-\bar{y}$ is given by

$$
\mathrm{SE}(\bar{x}-\bar{y})^{2}=\frac{(m-1) v_{x}^{2}+(n-1) v_{y}^{2}}{m+n-2}\left(\frac{1}{m}+\frac{1}{n}\right)
$$

under the assumption (8).

\section{Interval Estimation}

Let $M_{x}$ and $M_{y}$ be normally distributed populations with (8). Then, the statistic

$$
t=\frac{(\bar{x}-\bar{y})-\left(\mu_{x}-\mu_{y}\right)}{\operatorname{SE}(\bar{x}-\bar{y})}
$$

obeys a $t$ distribution with $m+n-2$ degrees of freedom. On the basis of this fact, we obtain the following confidence interval for $\mu_{x}-\mu_{y}$ :

$$
\bar{x}-\bar{y}-\delta^{\prime}<\mu_{x}-\mu_{y}<\bar{x}-\bar{y}+\delta^{\prime}
$$

with

$$
\delta^{\prime}=t_{m+n-2}\left(1-\frac{1}{2} \alpha\right) \operatorname{SE}(\bar{x}-\bar{y})
$$


where $t_{m+n-2}(1-\alpha / 2)$ denotes the $(1-\alpha / 2) \times 100$ th percentile of the $t$ distribution with $m+n-2$ degrees of freedom.

\section{Statistical Test}

The null hypothesis $H_{0}: \mu_{x}=\mu_{y}$ is tested by means of the confidence interval (11). A test with significance level $\alpha$ is implemented by the following rule:

Accept $H_{0}$ if and only if $\mu_{x}-\mu_{y}=0$ belongs to the interval (11), i.e., if and only if $|\bar{x}-\bar{y}|<\delta^{\prime}$ holds,

where $\delta^{\prime}$ is defined by (12). We refer to this test as a two-sample $t$ test.

Remarks. 1) A two-sample $t$ test is applied to unpaired samples. This means that the two samples must be independently chosen from two populations.

2) Suppose that we compare a set of data for patients obtained a year earlier with another set of data for the same patients obtained a month earlier. In this case, we cannot regard the sets of data as unpaired samples, because two results of a patient may have some dependence. These samples are called paired samples; the sizes of paired samples are the same $(m=n)$, and $x_{i}$ and $y_{i}$ make a pair. Paired samples should be analyzed in the form of differences $x_{i}-y_{i}$ by using the method stated in section 2. We refer to this test as a paired t test.

3) When we cannot assume that the populations are normally distributed, we should use nonparametric statistical methods as Wilcoxon rank sum test to compare (population) medians.

4) We can test the assumption (8) by an $F$ test using the statistic $F=v_{x}^{2} / v_{y}^{2}$. If we fail in accepting the hypothesis (8) as a result of the F test, Welch's test is available as an alternative of the two-sample $t$ test.

\section{Comparison of Two Populations: Probabilities}

In this section, we describe procedures to compare probabilities.

\section{Probabilities, Relative Risk, and Odds Ratio}

Suppose that two properties $X$ and $Y$ are defined on a population $M$. For example, $X$ means that a subject is exposed to a certain situation, and $Y$ means that we observe a certain outcome for a subject. We decompose $M$ into two subpopulations $M_{1}$ and $M_{2}$ according to whether $X$ holds or not, i.e., an element of $M$ belongs to $M_{1}$ (or $M_{2}$ ) if the element has (or does not have, resp.) the property $X$. Let $p_{1}$ and $p_{2}$ be the probabilities with which $Y$ may hold in $M_{1}$ and in $M_{2}$, respectively.

To compare $p_{1}$ and $p_{2}$, we introduce the following ratios:

$$
\begin{aligned}
& \text { relative risk }=\mathrm{RR}=\frac{p_{1}}{p_{2}}, \\
& \text { odds ratio }=\mathrm{OR}=\frac{p_{1} /\left(1-p_{1}\right)}{p_{2} /\left(1-p_{2}\right)} .
\end{aligned}
$$

If $X$ and $Y$ are statistically independent, we have $p_{1}=p_{2}$ and $\mathrm{RR}=\mathrm{OR}=1$.

\section{Point Estimation}

The population parameters $p_{1}, p_{2}, \mathrm{RR}$, and $\mathrm{OR}$ are estimated by using a randomly chosen sample. Sample data for this purpose are categorical and presented in the form of a contingency table (Table 1). The estimators of $p_{1}, p_{2}, \mathrm{RR}$, and $\mathrm{OR}$ are as follows:

$$
\begin{aligned}
& p_{1} \approx \frac{a}{a+b}, \quad p_{2} \approx \frac{c}{c+d}, \\
& \mathrm{RR} \approx \frac{a /(a+b)}{c /(c+d)} . \mathrm{OR} \approx \frac{a / b}{c / d} .
\end{aligned}
$$

\section{Interval Estimation}

We describe how to obtain confidence intervals of RR and of OR. Put

$$
\begin{gathered}
w=\sqrt{\frac{1}{a}-\frac{1}{a+b}+\frac{1}{c}-\frac{1}{c+d}}, \\
w^{\prime}=\sqrt{\frac{1}{a}+\frac{1}{b}+\frac{1}{c}+\frac{1}{d}} .
\end{gathered}
$$

Then, the confidence intervals of $\mathrm{RR}$ and $\mathrm{OR}$ with confidence level $1-\alpha$ are given by

$$
\begin{gathered}
\frac{a /(a+b)}{c /(c+d)} e^{-z(1-\alpha / 2) w}<\mathrm{RR}<\frac{a /(a+b)}{c /(c+d)} e^{z(1-\alpha / 2) w}, \\
\frac{(a / b)}{(c / d)} e^{-z(1-\alpha / 2) w^{\prime}}<\mathrm{OR}<\frac{(a / b)}{(c / d)} e^{z(1-\alpha / 2) w^{\prime}},
\end{gathered}
$$

respectively, where $z(1-\alpha / 2)$ denotes the $(1-\alpha / 2)$ $\times$ 100th percentile of the standard normal distribution and $e=2.71828 \cdots$ is a mathematical constant. 
Table 1 A contingency table: a sample consisting of $a+b+c+d$ elements is divided into four categories according to whether or not each element has the properties $X$ and $Y$, respectively.

\begin{tabular}{cccc}
\hline & with $Y$ & without $Y$ & Total \\
\hline with $X$ & $a$ & $b$ & $\mathrm{a}+b$ \\
without $X$ & $c$ & $d$ & $c+d$ \\
\hline Total & $a+c$ & $b+d$ & $a+b+c+d$ \\
\hline
\end{tabular}

\section{Statistical Test}

Let us consider statistical tests of the null hypothesis $H_{0}: p_{1}=p_{2}$ by the sample data shown in

\section{Table 1.}

The simplest procedure to test $H_{0}$ is a chi-square test (or Fisher's exact test) for a contingency table. More sophisticated tests rely on the formulae (19) and (20). For a test using RR (or OR) with significance level $\alpha$, we accept $H_{0}$ if and only if the value $R R=1$ (or $\mathrm{OR}=1$ ) satisfies (19) (or (20), resp.).

Remarks. 1) Interval estimations of relative risk and of odds ratio are useful for assessing causal relations between exposures and outcomes in cohort studies and case-control studies. To confirm statistical associations (instead of causal relations) in crosssectional studies, the chi-square test is usually used.

2) In a case-control study, we cannot estimate relative risk by (16). In fact, the ratios $a: b:(a+b)$ and $c: d:(c+d)$ are determined by the study plan (instead of the populations), hence they do not reflect objective properties of the populations. In this case, the odds ratio should be estimated, because the ratios $a: c$ and $b: d$ have objective meanings and odds ratio approximates relative risk if $p_{1}$ and $p_{2}$ are small, as is seen from (13) and (14).

3) Matched-pair data in a case-control study may have some dependence within the pair. We therefore cannot regard the data as being independently chosen from two populations $M_{1}$ and $M_{2}$. In this case, McNemar's test can be used to test the null hypothesis $p_{1}=p_{2}$.

\section{Multiple Comparisons}

In this section, we deal with the problem of comparing means of three or more populations. It is essential to understand the reason why pairwise comparisons may cause a trouble.

\section{Successive Applications of Statistical Tests}

Let $T_{1}$ and $T_{2}$ be two tests of a null hypothesis $H_{0}$. We compose them into a test $T$ as follows: 1) perform $T_{1}$ with significance level $\alpha$;) perform $T_{2}$ with significance level $\alpha$; and 3) reject $H_{0}$ if at least one of these tests rejects $H_{0}$. The key point is that the overall significance level $\alpha^{\prime}$ of the test $T$ is not $\alpha$. In fact, if $H_{0}$ is true, $H_{0}$ may be rejected with probability $\alpha$ in each of $T_{1}$ and $T_{2}$, hence the probability $\alpha^{\prime}$ with which the test $T$ may reject $H_{0}$ is given by

$$
\alpha^{\prime}=1-(1-\alpha)^{2}=2 \alpha-\alpha^{2}(>\alpha)
$$

where we have assumed that consequences of $T_{1}$ and $T_{2}$ are independent. Although it is possible to set $\alpha$ so that $\alpha^{\prime}$ may be a desired value, e.g., 0.05 , the problem becomes serious, when we successively perform many tests, because $\alpha$ should be set extremely small.

For the same reason, pairwise comparisons may cause an undesired change of significance level, when we compare three or more populations. In what follows, we discuss a solution to this problem.

\section{Comparison of Means of Three or More} Populations

Suppose that we are comparing means of $r$ populations $M_{1}, M_{2}, \cdots, M_{r}$. Denote the mean and variance of $M_{i}$ by $\mu_{i}$ and $\sigma_{i}^{2}$, respectively, for $i=$ $1,2, \cdots, r$, and assume

$$
\sigma_{1}^{2}=\sigma_{2}^{2}=\cdots=\sigma_{r}^{2}
$$

To test the null hypothesis $H_{0}: \mu_{1}=\mu_{2}=\cdots=\mu_{r}$, we randomly choose samples from the populations (Table 2). Let $n_{i}$ (or $\bar{x}_{i}$ ) be the size (or the sample mean, resp.) of the sample $x_{i 1}, x_{i 2}, \cdots$ chosen from $M_{i}$ for $i=1,2, \cdots, r$, and denote the total size by $N=n_{1}$ $+n_{2}+\cdots+n_{r}$. Then, the sample mean over all groups is given by

$$
\bar{x}=\frac{1}{N} \sum_{i} \sum_{j} x_{i j}=\frac{1}{N} \sum n_{i} \bar{x}_{i} .
$$

We introduce two kinds of sums of squares: 
Table 2 A set of samples for a one-way ANOVA: a sample chosen from the population $M_{i}$ consists of $n i$ elements and has sample mean $\bar{x}_{i}$. The total size of the samples is $N$, and the sample mean over all groups is $\bar{x}$.

\begin{tabular}{cccc}
\hline Population & Sample & $\begin{array}{c}\text { Sample } \\
\text { size }\end{array}$ & $\begin{array}{c}\text { Sample } \\
\text { mean }\end{array}$ \\
\hline$M_{1}$ & $x_{11}, x_{12}, \cdots \cdots$ & $n_{1}$ & $\bar{x}_{1}$ \\
$M_{2}$ & $x_{21}, x_{22}, \cdots \cdots$ & $n_{2}$ & $\bar{x}_{2}$ \\
$\vdots$ & $\vdots$ & $\vdots$ & $\vdots$ \\
$M_{r}$ & $x_{r 1}, x_{r 2}, \cdots \cdots$ & $n_{r}$ & $\bar{x}_{r}$ \\
\hline Total & & $N$ & $\bar{x}$ \\
\hline
\end{tabular}

within-Groups Sum of Squares:

$$
S S_{\text {within }}=\sum_{i} \sum_{j}\left(x_{i j}-\bar{x}_{i}\right)^{2} \text {, }
$$

Between-Groups Sum of Squares:

$$
S S_{\text {between }}=\sum_{i} n_{i}\left(\bar{x}_{i}-\bar{x}\right)^{2} \text {, }
$$

and put

$$
F=\frac{\frac{1}{r-1} S S_{\text {between }}}{\frac{1}{N-r} S S_{\text {within }}} .
$$

Since the quantity $S S_{\text {within }}$ measures the variances of data within groups, $S S_{\text {within }}$ is insensitive to the population means $\mu_{1}, \mu_{2}, \cdots, \mu_{r}$, whereas $S S_{\text {between }}$ that measures the variance of data between groups is sensitive to population means and is likely to be large unless

$$
\mu_{1}=\mu_{2}=\cdots=\mu_{r}
$$

holds. Then, we expect that $F$ may tend to be large unless (27) holds.

Assume that $M_{1}, \quad M_{2}, \cdots, M_{r}$ are normally distributed populations with (22) and (27). Under these assumptions, $F$ obeys an $F$ distribution with $r$ -1 and $N-r$ degrees of freedom. Therefore, in the situation where (22) has been confirmed, the null hypothesis $H_{0}: \mu_{1}=\mu_{2}=\cdots=\mu_{r}$ is tested with significance level $\alpha$ by the following rule:

Reject $H_{0}$ if and only if $F>F_{N-r}^{r-1}(1-\alpha)$, where $F_{N-r}^{r-1}(1-\alpha)$ denotes the $(1-\alpha) \times 100$ th percentile of the $F$ distribution with $r-1$ and $N-r$ degrees of freedom. Note that this is a one-sided test. If the null hypothesis $H_{0}$ is false, the statistic $F$ is likely to be large, hence we should reject $H_{0}$ if and only if $F$ is large.
The above procedure is called a one-way analysis of variance (abbreviated as a one-way ANOVA). In a situation where the population $M$ is decomposed into subpopulations $M_{1}, M_{2}, \cdots, M_{r}$ according to some factor, e.g., age of subjects, one-way ANOVA works as a test of whether or not the factor has an effect on outcomes.

Remarks. 1) As long as (22) is satisfied, the oneway ANOVA is also available in the situation where the distributions of the populations may slightly deviate from a normal distribution. In this sense, ANOVA is robust. However, if the distributions of populations are far from being normal, we should use a nonparametric alternative called the KruskalWallis test.

2) To confirm the assumption (22) is a subtle problem. For this purpose, Bartlett's test is known. As an alternative of the one-way ANOVA which is available without the assumption (22), an approximate test using weighting is known. However, from a practical viewpoint, it may be advisable to plan a study so that sample sizes $n_{1}$, $n_{2}, \cdots, n_{r}$ are equal or close to equal, because the assumption (22) is not relevant in this situation.

3) Even if we succeed in rejecting the null hypothesis $H_{0}: \mu_{1}=\mu_{2}=\cdots=\mu_{r}$, we cannot clarify which population mean deviates. This problem needs a further analysis, which will be discussed in the next subsection.

4) Using the one-way ANOVA, we can study an effect of a factor on outcomes. To assess effects of two factors, we should use a two-way ANOVA.

\section{Contrasts}

To compare three means $\mu_{1}, \mu_{2}$, and $\mu_{3}$ in detail, we may want to perform multiple comparisons, i.e., inferences of three differences $\mu_{1}-\mu_{2}, \mu_{1}-\mu_{3}$, and $\mu_{2}$ - $\mu_{3}$. It will be convenient to introduce a linear combination $c_{1} \mu_{1}+c_{2} \mu_{2}+c_{3} \mu_{3}$ as a generalization of the differences, where $c_{1}, c_{2}$, and $c_{3}$ are constants satisfying $c_{1}+c_{2}+c_{3}=0$.

A linear combination

$$
\lambda=c_{1} \mu_{1}+c_{2} \mu_{2}+\cdots+c_{r} \mu_{r}
$$

of $r$ means $\mu_{1}, \mu_{2}, \cdots, \mu_{r}$ is called a contrast, if 


$$
c_{1}+c_{2}+\cdots+c_{r}=0
$$

holds. In what follows, we discuss how to infer the value of $\lambda$ from the sample shown in Table 2 .

Because $\mu_{i}$ has an estimator $\bar{x}_{i}$, the value of $\lambda$ is estimated by the statistic

$$
L=c_{1} \bar{x}_{1}+c_{2} \bar{x}_{2}+\cdots+c_{r} \bar{x}_{r}
$$

Under the assumption (22), L has the squared standard error $\mathrm{SE}(L)^{2}$ given by

$$
S E(L)^{2}=\frac{1}{N-r}\left(\frac{c_{1}^{2}}{n_{1}}+\frac{c_{2}^{2}}{n_{2}}+\cdots+\frac{c_{r}^{2}}{n_{r}}\right) S S_{\text {within }}
$$

where $S S_{\text {within }}$ is defined by (24).

Assume that population $M_{1}, M_{2}, \cdots, M_{r}$ are normally distributed and (22) holds. Then, the statistic

$$
t=\frac{L-\lambda}{\mathrm{SE}(L)}
$$

obeys a $t$ distribution with $N-r$ degrees of freedom. A $t$ test by means of (32) being possible, its successive applications may cause a problem, in particular if we explore various contrasts one after another. The key point of the solution is to take into account all contrasts $\lambda$ with (29) instead of looking only at the ones that have direct relevance to our interest. Let $F_{N-r}^{r-1}(1-\alpha)$ be the $(1-\alpha) \times 100$ th percentile of an $F$ distribution with $r-1$ and $N-r$ degrees of freedom, and put $\rho=(r-1) F_{N-r}^{r-1}(1-\alpha)$. Then, the following event occurs with probability 1 $-\alpha$ :

the inequality $t^{2}<\rho$ holds for any contrast $\lambda$,

where $t$ is defined by (32). Note that the inequality $t^{2}$ $<\rho$ yields the following interval estimation of the corresponding contrast $\lambda$ :

$$
L-\sqrt{\rho} \cdot S E(L)<\lambda<L+\sqrt{\rho} \cdot S E(L) .
$$

The confidence level is not reduced (from $1-\alpha$ ), no matter how many contrasts we consider, because $t^{2}<\rho$ holds for any contrast (with probability $1-\alpha$ ). We refer to this method as Scheffé's procedure.

As an example, we compare three means $\mu_{1}, \mu_{2}$, and $\mu_{3}$. Consider three contrasts and their estimators

$$
\lambda_{1}=\mu_{1}-\mu_{2}, \lambda_{2}=\mu_{1}-\mu_{3}, \lambda_{3}=\mu_{2}-\mu_{3},
$$

$$
L_{1}=\bar{x}_{1}-\bar{x}_{2}, L_{2}=\bar{x}_{1}-\bar{x}_{3}, L_{3}=\bar{x}_{2}-\bar{x}_{3} .
$$

The corresponding $t$ statistics are

$$
t_{i}=\frac{L_{i}-\lambda_{i}}{\mathrm{SE}\left(L_{i}\right)}, i=1,2,3,
$$

and the following event occurs with probability larger than $1-\alpha$ :

the inequalities $t_{i}^{2}<\rho$ simultaneously hold for $i=1$, $2,3$.

Because we consider only three contrasts (34) instead of all the contrasts, the probability is larger than $1-\alpha$. The inequality (33) yields simultaneous confidence intervals

$$
L_{i}-\sqrt{\rho} \cdot \mathrm{SE}\left(L_{i}\right)<\lambda_{i}<L_{i}+\sqrt{\rho} \cdot \mathrm{SE}\left(L_{i}\right), \quad i=1,2,3
$$

for $\lambda_{1}, \lambda_{2}$, and for $\lambda_{3}$ with confidence level larger than $1-\alpha$. By means of (37), we can determine which contrasts differ from 0 . Suppose we find that $\lambda_{1}>0$, $\lambda_{2}>0$, and $\lambda_{3}=0$. Then, our conclusion is $\mu_{1}>\mu_{2}=$ $\mu_{3}$.

Remarks. 1) We can apply Scheffé's procedure to the contrast $\lambda=2 \mu_{1}-\mu_{2}-\mu_{3}$ for comparison of $\mu_{1}$ and the average of $\mu_{2}$ and $\mu_{3}$. More complicated comparisons are possible using contrasts.

2) The actual confidence level of the confidence intervals (37) is larger than $1-\alpha$. This means that Scheffé's procedure yields conservative results.

3 ) If the sizes $n_{1}, n_{2}, \cdots, n_{r}$ of samples are the same, the Tukey test is available for multiple pairwise comparisons.

\section{References}

1. Watanabe H: Applications of Statistics to Medical Science, I. Journal of Nippon Medical School 2011; 78: 274-279.

2. Rosner B: Fundamentals of Biostatistics, 7th ed. 2011; Brooks/Cole, Boston.

3. Armitage P, Berry G, Matthews JNR: Statistical Methods in Medical Research, 4th ed. 2002; Blackwell Science, Massachusetts.

(Received, October 24, 2011)

(Accepted, December 7,2011) 